\title{
User-centered personas for PhysPort
}

\author{
Adrian M. Madsen, Sarah B. McKagan \\ American Association of Physics Teachers, 1 Physics Ellipse, College Park, MD 20740 \\ Linda E. Strubbe, Eleanor C. Sayre, Dina Zohrabi Alaee, Tra Huynh \\ Department of Physics, Kansas State University, Manhattan, KS 66506
}

\begin{abstract}
PhysPort is a professional development website for physics faculty to develop their teaching through research-based resources. As part of PhysPort's ongoing research efforts, we conducted interviews with 23 physics faculty from diverse instructional and institutional contexts in the US. From our interviews, we sought common experiences, motivations, and pain points to develop personas - person-like constructs - of physics faculty in the US. Our research focuses on the perspectives of the key users of our site, and thus we take a user-centered perspective rather than a researcher-centered perspective. We developed personas, which are person-like constructs that are developed based on salient characteristics of actual users, that enable designers to create resources to meet actual user needs without designing for the idiosyncrasies of specific users. We present our set of six personas of physics faculty members: a faculty member who is new to improving his teaching; one who takes up his department's practices; one who wants her teaching to feel good; one who is comfortable in her teaching; one who is continuously improving; and one who solves big problems in her department. These personas of physics faculty making changes to their teaching can be used more broadly to improve the design and development of professional development resources and activities for physics faculty.
\end{abstract}




\section{INTRODUCTION}

In order to support faculty well through professional development activities and resources, it is important to design these activities and resources to meet the varied needs of real faculty and align with their actual motivations and attitudes. This is in contrast to the activities and resources being designed based on the designers' best guesses about what their users need or the designers' own preferences or opinions. This idea is supported by the Increase the Impact report [1] which explains a key step to designing educational innovations for sustained adoption is identifying your adopters, understanding who they are and why they might want to use your product. One way to do this is by using personas.

Personas are person-like constructs that are developed based on salient characteristics of actual users. They package a large amount of information into a succinct format that is easy to understand and gives the designers something concrete to discuss $[2,3]$. Interview data from a diverse group of potential users is synthesized into these archetypes of people (Figure 1). A persona doesn't represent any single person, but is very person-like, enabling designers to design for a "person" without designing for idiosyncratic details of an actual person. Usually a group of personas is created to represent the key types of users of a resource. Personas also help designers prioritize who they are designing different features of their resource for, instead of just designing for a generic user. The abstraction of personas away from the interviewees upon which they are based ensures the anonymity of participants. Finally, personas are referred back to throughout the entire project to ensure that the resource doesn't depart from the needs of actual users.

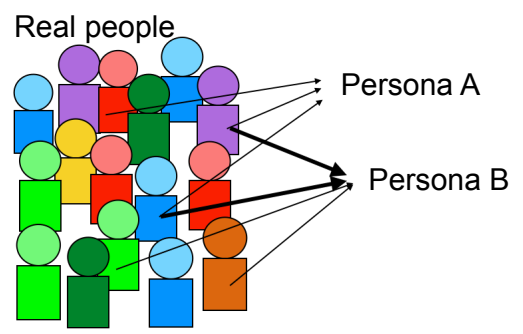

FIG. 1. Illustration of how characteristics of many real people are rearranged to develop a set of personas.

We developed a set of personas as part of the larger PhysPort research endeavor focusing on developing resources to support physics faculty in developing their own teaching. PhysPort (http://physport.org) is a website that supports physics faculty with research-based teaching and assessment in their classes. PhysPort was created over time, as various pieces of the site were funded, and now the team is working to redesign the site to make it a more coherent whole. The design problem for the PhysPort redesign is, "How can physics faculty find what they need on PhysPort to make changes to their teaching?"

In this paper we discuss the method we used to create personas of our users and present our resulting personas. We also discuss how these personas can be used more broadly in professional development for physics faculty beyond PhysPort.

\section{METHODS}

We used phenomenographic semi-structured interviews to learn about how instructors approached making changes to their teaching [4]. A secondary aim was to develop personas of PhysPort users. We choose to focus on how faculty make changes to their teaching instead of asking faculty about their teaching in general, because we think that faculty are far more likely to look for professional development resources when they are thinking about making changes to their teaching. We conducted video interviews remotely, each of which lasted about 1 hour. During the interview, instructors were asked to describe their instructional practices: how they approach their teaching; what kinds of changes they were making to their teaching; their motivation to make changes; their assessment practices around the change; resources they use and how they use them; and challenges they experienced with their teaching. We also asked about their background, departmental culture, collaboration around teaching, and their ethnicity and gender. After several interviews, the protocol was updated to probe themes around motivation and development of teaching practices more carefully.

We wanted to interview instructors at US institutions who had recently made changes to their teaching (big or small) or were planning upcoming changes. We recruited instructors from different sources. To ensure diversity in our data set, we solicited faculty with a range of years of teaching experience, from different types of institutions and departments, and with different types of appointments. Twenty-three physics instructors were interviewed. Participants had a range of teaching experience: 15 had less than 10 years of experience, 8 had more than 11 years of experience. There were 10 participants at private institutions and 13 at public institutions. There were 10 participants at doctoral granting institutions, 7 at masters granting institutions, 4 at baccalaureate granting institutions and 2 at associates granting institutions. We interviewed seven women and sixteen men, and 5 people of color and 18 white people. We interviewed 3 Full Professors, 4 Associate Professors, 10 Assistant Professors and 6 who held titles of Instructors or Lecturer or Teaching Professor. All interviews were video recorded and transcribed using a paid service.

\section{ANALYSIS}

We first completed a phenomenographic analysis [4] 
of the interviews to produce a set of categories which described the themes that emerged in the interviews [4]. This process involved immersion in data, category development and refinement, connecting categories to specific quotes, coding all quotes from interviews using categories, and developing robust descriptions of categories. For a full description of this process and results see Zohrabi Alaee et al., 2019 [5]. The categories that resulted from this analysis included faculty motivations and attitudes around making changes to their teaching, types of instructional practices they are trying, ways they decide something new is working, resources they are using or want and challenges that they experience.

We used the list of detailed sub-categories for the faculty motivations and attitudes around making changes to teaching as the basis for our personas. Several team members worked together to assemble the sub-categories for motivations and attitudes into person-like constructs, or personas. In this process, team members proposed different personas of faculty, and described their motivations and attitudes around making changes to their teaching, things they try in their teaching, how they know if they work, resources they use and what resources they need. Together, the team expanded some of personas into more than one, and collapsed other personas together. After an initial meeting, one team member went back to the interview transcripts and notes to compare the personas to the actual faculty interview data. She created a list of quotes from the transcripts that illustrated each of the personas, and found many ways to revise the personas to more clearly reflect the interview data. The team met again and negotiated these changes. For example, one persona that was initially proposed was motivated to try a new thing in their teaching because they needed tenure or to keep their job. After reviewing the transcripts, the team found that getting tenure or keeping their job wasn't a primary motivation for any of the faculty interviewed. Using the quotes from the transcripts, the team revised the personas to address this.

After this, the lead author went back to the interview data again to categorize each faculty members' primary and secondary personas. She found that several faculty weren't well represented in the personas, as well as ways to revise the existing personas to better match the interview data. The team discussed these findings, and made further changes to the personas. During the persona development process, the team members were committed to having a positive, asset-based view of faculty.

After settling on the major details of the personas, we added more details to make the personas feel more personlike. These details include their picture, particular name, gender, ethnicity, institution type, and years of experience. The number of years of experience for a given persona was roughly based on the experience of the actual faculty represented by this persona. We have found that including a short description of the persona and a first name that starts with the same letter as the descriptor makes it easier to remember and talk about the personas. We also believe its important to present a diverse set of physics faculty personas, to illustrate that all kinds of people can be physics faculty. To come up with the particular names for each persona, two members used an online pseudonym generator and chose a diverse set of names that are easy for Americans to pronounce. We sought to avoid gender or ethnic stereotypes when assigning identities to different personas. We then shared our named personas and descriptions with colleagues, and renamed personas when issues came up.

\section{FINDINGS}

Our analysis process resulted in six personas representing physics faculty making changes to their teaching. We describe each below and in Table 1 .

Claude, the "cautious implementer" is an assistant professor who is new to improving his teaching. He is teaching in a large department, at a 4-year regional institution and has been there for 2 years. He heard about active learning teaching strategies as a teaching assistant during graduate school, and through a professional development conference he attended. Claude feels like he should move away from pure lecture, and try some new active learning strategies to help his students learn, but is worried that some strategies are too radical and won't help. He is also worried that these new strategies won't work, students will be unhappy and not learn well and he won't be able to cover all that he should. He is willing to try small incremental changes to his teaching, especially changes that don't take much time. If something is too time intensive, he feels he won't be able to do it. Claude will know that the new teaching strategies worked if his students are learning the content and seem to like the activity. Currently Claude uses ideas he learned from a national teaching workshop, discussions with his colleagues, and ideas he comes up with himself. Claude wants simple explanations of short timescale strategies to try, and troubleshooting help including explanations of what might go wrong and how to address it, as well as help after he tries something new to address challenges that he faced.

Diego, the "departmental participant", is an assistant professor who is also relatively new to teaching. He has been in his current large department at an R1 university for 3 years. His department has a strong culture and set of practices around teaching, and shared teaching materials. Diego wants to take up his departments' teaching practices, but there is also an expectation that he should do so. Diego feels like learning to teach with the active learning teaching methods his department uses was hard at first, but it gets better. He was nervous to begin with, but now he loves it. His department is happy when he uses the common teaching methods and materials, and students do well on metrics that Diego's colleagues find important. 
TABLE 1. Key quotes, descriptions and key goals of our six personas of physics faculty making changes to their teaching.

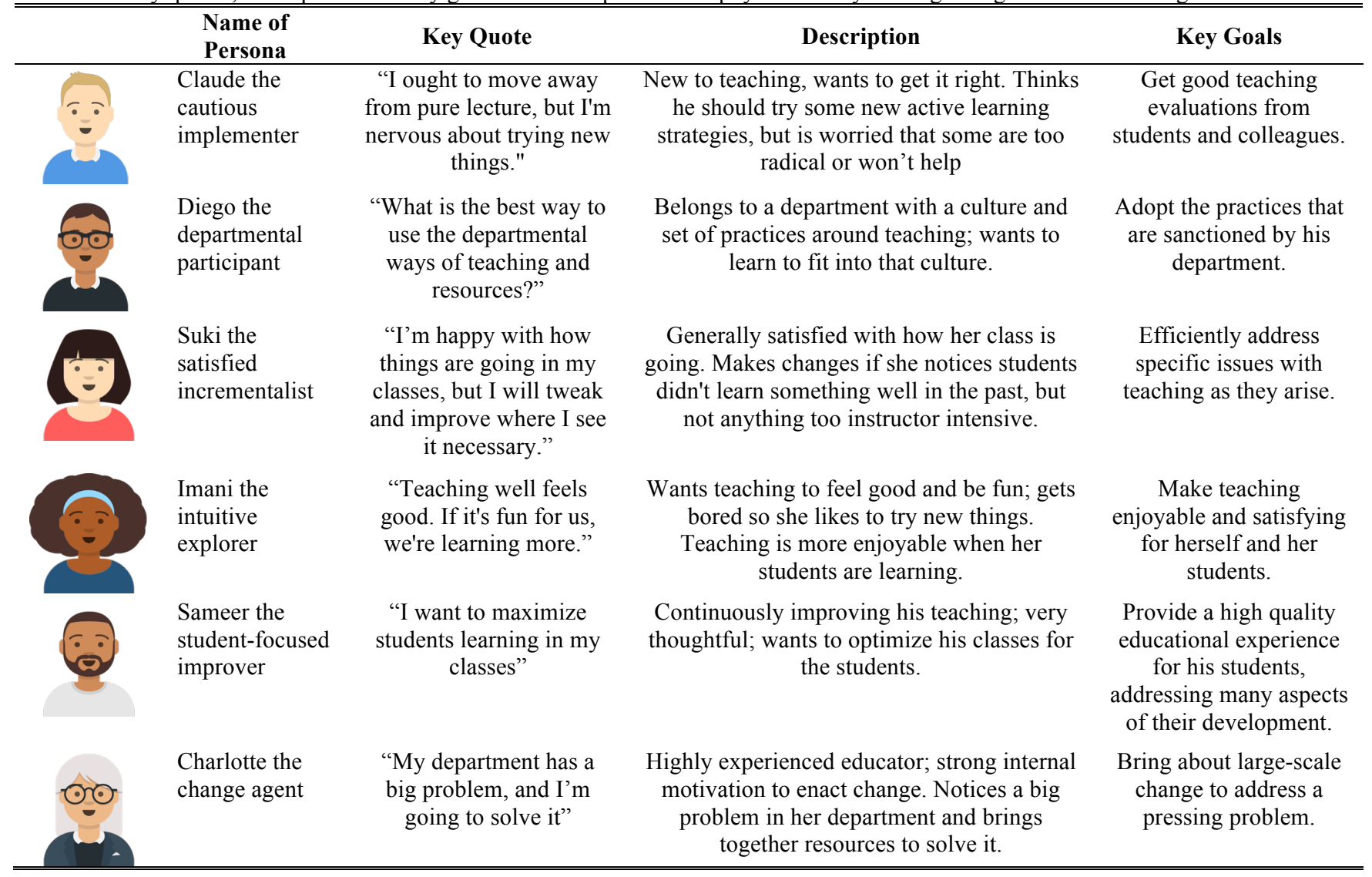

Diego primarily uses teaching resources he gets in coteacher meetings and ideas from informal conversations with faculty in his department. He also gets some ideas and materials from workshops at his campus teaching and learning center, and materials that came with his textbook. Diego wants more resources and training around the teaching practices his department already uses or resources are compatible with his departmental practices.

Suki, the "satisfied incrementalist", is an associate professor with 12 years of experience in her small department at a community college. In general, she is happy with how her class is going. If she's going to make a change to her teaching it is because she noticed her students didn't learn something very well in the past. But Suki may avoid or abandon any teaching changes that are too time intensive for her, as she doesn't have much extra time to devote to teaching. Suki only changes a few things each term (incrementalist). For example, in a given term she will write a couple of new activities or change the structure of an existing activity to address the conceptual problems her students had last year. She assesses the effectiveness of her change by looking at how her students learned the content this year as compared to last. Suki has developed a corpus of teaching ideas to try as a result of many professional development experiences, and what she learned as a teaching assistant in graduate school. Suki wants more extensive help with and teaching materials for a certain topic that students didn't do well on last term.

Imani, the "intuitive explorer", is a full professor with 8 years of experience in her medium-sized department at a liberal arts college. Imani cares a lot about her own affect when teaching and wants her teaching to feel good. It's more enjoyable for her when students are learning and if the class is fun for her and the students, she feels they are learning more. She is often bored with how she's currently teaching, and wants to try something new. Imani is happy to try new things if they are going to make her class feel better. The changes she is interested in are usually incremental and in combination with other things. Imani is not too worried about the provenance of each new idea. She really likes clever teaching ideas (e.g. a new way to explain a concept) and likes to think about trying them. She feels she can read her class and tell if the students are getting it. Imani has informal chats with colleagues to get new ideas. She also dreams up new things to try in her class herself and occasionally sees a talk, or reads an article and gets new teaching ideas from it. Imani wants ideas for new ways to teach that will feel good to her. These could be small grain size, or large pedagogical changes. When developing resources for Imani its important to highlight the positive instructor and student affect that they enable. 
Sameer, the "student-focused improver", is an assistant professor with 6 years of experience in his small department at a 4-year regional college. He cares deeply about his students' learning and development including their skills, content knowledge, affect, identity, etc. He also believes that keeping his students engaged will support their learning. Sameer is continually working on improving his teaching and is really thoughtful about his class. He will try new things in his teaching that take him a lot of time and are hard for him if he thinks that they will benefit his students. Sameer always wants to add new teaching strategies to what he already does. He is open to new big ideas and would make big changes to his teaching if he thought they would help students. He also wants to broaden his perspective on important issues that influence his students/classroom, such as issues of equity and inclusion. Sameer believes that it is important to gather evidence of his students learning and growth, and uses a combination of student evaluations, exams, standardized research-based assessment instruments as well as informal student feedback. He uses any and every resource he can find to improve his teaching, including books about teaching, journal articles about education research, professional development workshops locally and nationally, conversations with local and remote colleagues etc. Sameer wants ideas on things to try in his class, or issues to be aware of. He wants detailed information, in order to use them in his class soon. He also wants to know about the evidence for why these work.

Charlotte, the "change agent", is a full professor who has been at her current large department at an R1 University for 20 years. Charlotte has noticed a big problem in her department such as a high Drop/Fail/Withdraw (DFW) rate or low learning gains in introductory classes, and wants to solve it. Charlotte recognizes that solving this big problem is hard work but very important. She also sees that she needs to build support in her department to do this, possibly relying on grant funding. Charlotte is open to major pedagogical or programmatic changes that involve multiple faculty or courses to solve this big problem. She will know that she is successful if her big problem is solved. Charlotte looks into physics education research and more general education research literature to find the most effective solution to her problem. She also consults with experts to get personalized help. Charlotte wants curated explanations of how to solve her department's problem, with links to results about the solution and recommendations on experts to talk to.

\section{DISCUSSION AND IMPLICATIONS}

These personas of physics faculty making changes to their teaching enable designers of professional development for physics faculty to understand, empathize with and prioritize different types of physics faculty as they create different features of their resource. These specific personas enable professional development designers in physics to be "user-centered" instead of researchercentered, since they were developed based on physics faculty member's internal motivations and attitudes around making changes to their teaching.

This set of personas is not only useful for redesigning PhysPort, but you too could use these personas when developing professional development for physics faculty. Each of these personas has unique motivations, and needs, and you can customize your resource/activity to appeal to and meet these. For instance, if you are developing research-based teaching materials that you hope other physics faculty will use, you can think about how your teaching materials would align with the motivations of each of these personas. You can also choose which of these personas you most want to support with your materials, and make sure you explicitly design your materials for those personas. For example, Claude, the "cautious implementer" would be more comfortable using new materials in small pieces, so he can have small successes. He would also like troubleshooting support if he has trouble with something he is trying. On the other hand, teaching materials you develop for Charlotte the "change agent" should target a big problem that she cares about. Charlotte is willing to make big changes to solve her problem, so you would want to offer her a whole suite of resources and expert consultation. She wants help not just with implementing the materials, but also with gathering evidence showing that her big problem is solved. Imani the "intuitive explorer" is interested in teaching materials that will make her course more enjoyable. When developing materials for Imani, include information about how faculty and students enjoy them.

Notably, there is no "skeptic" persona of faculty making changes to their teaching. This is because most faculty who do not use teaching methods/materials developed by physics education researchers do not consider themselves to be skeptics. Instead they view themselves as agentic faculty members using their own expertise and following their own motivations, to teach in the way they find best. In labeling these faculty members as "skeptic" we are only looking at them in relationship to ourselves as physics education researchers. Since these personas are developed based on interview accounts of faculty members' own experiences of teaching, they reflect the faculty members' perspective, instead of the researchers' perspective. This enables researchers/designers to avoid pitfalls like thinking of users in relation to themselves, and designing resources, for example, to convince skeptics, when the faculty who they are designing for don't think of themselves in this way.

\section{ACKNOWLEDGMENTS}

Thank you to the faculty who participated in our interviews. This work is supported by NSF grant \#1726113. 


\section{References}

[1] C. Henderson, R. Cole, J. Froyd, D. Friedrichsen, R. Khatri, and C. Stanford, Designing Educational

Innovations for Sustained Adoption: A How-to Guide for Education Developers Who Want to Increase the Impact of Their Work (Increase the Impact, Kalamazoo, MI, 2015).

[2] A. Yström, L. Peterson, B. Von Sydow, and J. Malmqvist, in Proc. 6th Int. CDIO Conf. (Montréal, 2010).

[3] J. Pruitt and T. Adlin, The Persona Lifecycle: Keeping People in Mind Throughout Product Design (Morgan Kaufmann, Burlington, 2010).

[4] F. Marton, J. Thought 21, (1986).

[5] D. Zohrabi Alaee, L. E. Strubbe, E. C. Sayre, A. M. Madsen, and S. B. McKagan, Phys. Rev. Phys. Educ. Res. in prep, (2019). 